\title{
Assessing the strength of in situ Portland cement concrete by internal fracture tests*
}

\author{
A. J. Chabowski and D. W. Bryden-Smith \\ Contribution by J. H. Bungey \\ University of Liverpool : Department of Civil Engineering
}

Dr Chabowski and Mr Bryden-Smith have produced valuable data confirming and enhancing the value of the test approach they discuss. The significant feature of this method is that, if it is properly executed, an estimate of in situ concrete strength can be obtained from a single generalized calibration curve for natural aggregates. Although limited to the surface zone, this is something that cannot justifiably be claimed for any other non-destructive or semidestructive method. The principal application is therefore most likely to be as an alternative to cores in situations where these cannot easily be obtained, although the accuracy of strength prediction is inferior even to that of small-diameter cores ${ }^{(1)}$.

A recently published account ${ }^{(2)}$ of work in this area has demonstrated that a mechanical direct-pull loading method, a version of which is shown in Figure I, offers greater sensitivity of load application and measurement than the torque meter. The resulting reduction of test variability means that an improved accuracy of strength prediction is possible and has enabled a clear distinction in test variability to be established between 10 and $20 \mathrm{~mm}$ aggregate concrete. Furthermore the importance of careful control of load application rate must not be overlooked, and techniques involving 'settling pauses' were found to yield less consistent results than steady loading application. It is particularly important that a calibration appropriate to the loading method is used;-this cannot be over-emphasized, since the authors' curves have been observed in use with a totally different hydraulic loading method by a commercial organization carrying out testing in situ.

I agree with Dr Chabowski and Mr Bryden-Smith

*Pages 164-172 of MCR 112. that the effect of compressive stress upon the magnitude of measured pull-out force is small. Tests on $4.8 \mathrm{~m} \times 500 \mathrm{~mm}$ deep beams under load suggest, however, that the coefficient of variation of test results will show a marked increase under even low levels of either compressive or tensile stress. This is

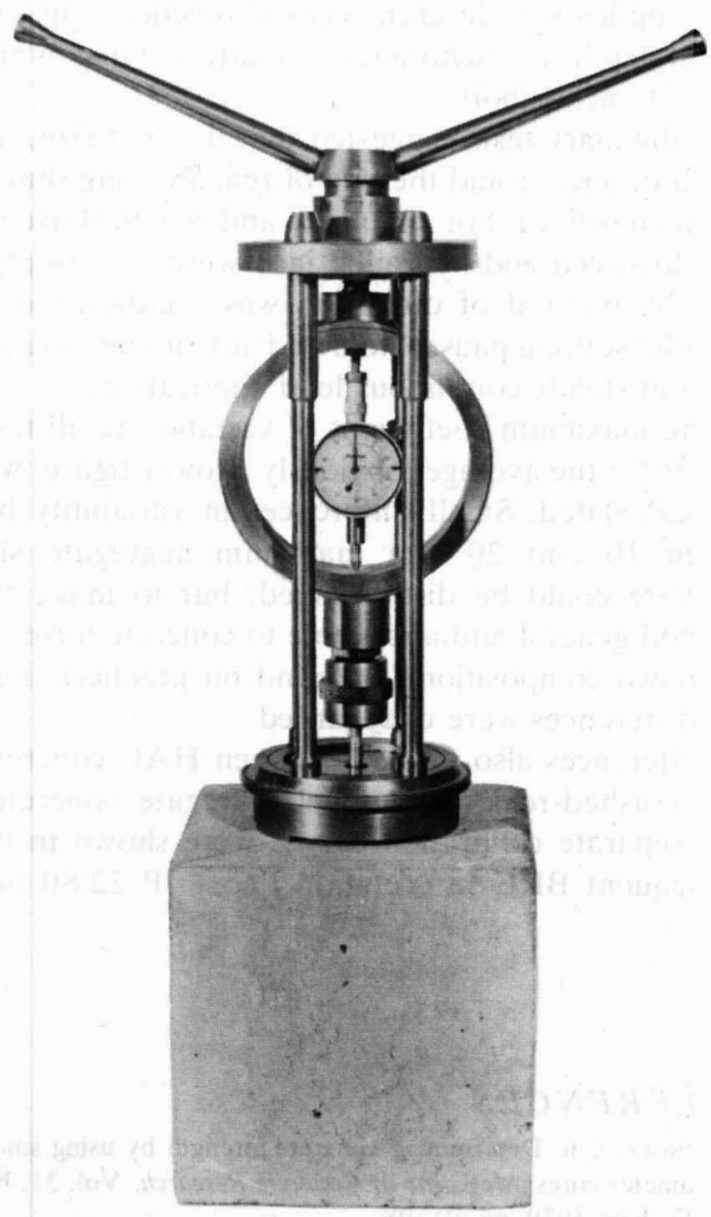

Figure I: Equipment for mechanical direct-pull tests. 
T A B LE 1: Variability of pull-out tests using direct-pull equipment.

Average coefficient of variation

(\%)

\begin{tabular}{c|c|c}
\hline Cubes & Unloaded beams & Loaded beams \\
\hline $5 \cdot 4$ & $8 \cdot 6$ & $12 \cdot 2$ \\
\hline
\end{tabular}

indicated in Table $\mathrm{I}$, which relates to $10 \mathrm{~mm}$ North Notts gravel concrete in cubes and at individual levels of beams, which were tested unloaded and again when under load. Tests on cubes of similar concrete using torque meter equipment yielded an average coefficient of variation of $12.4 \%$, and it would thus be interesting if the authors could provide information concerning the variability of the measured torque values of their tests on both cubes and stressed specimens. This may be of importance in the interpretation of the results of in situ tests, since their quoted accuracies of strength prediction appear to be based on the results of tests on cubes. It may be that the number of tests necessary to achieve comparable accuracies should be increased for members tested in service.

\section{Reply by authors}

We thank Mr Bungey for his contribution and would like to reply as follows.

The internal fracture test equipment was developed for ease of application in restricted areas on horizontal surfaces on top of and underneath concrete elements and on vertical surfaces of columns. Development work on the test included use of equipment with a load-cell, hydraulic jack, continuous load application, different sizes of reaction ring, different depths of anchorages and different makes and sizes of anchor bolt.

Preliminary tests suggested that the bolt size, the depth of anchor and the size of reaction ring should be standardized. For simplicity and practical use on site, load-cell and hydraulic jack were not adopted and the method of operation was standardized to include 'settling pauses' to avoid any timing requirement in steady continuous load application.

The maximum coefficient of variation in all tests was $16 \%$; the average, obviously a lower figure, was not calculated. Small differences in variability between 10 and $20 \mathrm{~mm}$ maximum aggregate size concrete could be distinguished, but to make the method general and applicable to concrete mixes of unknown composition (as found on practical sites) the differences were disregarded.

Differences also existed between HAC concrete, and crushed-rock and gravel-aggregate concretes, and separate calibration curves were shown in the subsequent BRE Information Paper IP 22/80 but,

because the effect was relatively small, the average relationship was recommended for practical use.

The effect of compressive stress upon the results can be ignored provided that locations for test are chosen where compressive stresses are low - say, less than $4 \mathrm{~N} / \mathrm{mm}^{2}$ - otherwise a correction of $1 \%$ of estimated cube strength for each $1 \mathrm{~N} / \mathrm{mm}^{2}$ of compression is suggested. Higher precompression cannot be ignored but, since the scatter of results found in the experimental work was considerable, the results were shown in full in the paper to allow engineers to make their own decisions.

The graphs were based on tests on cubes, because cubes provided a means of testing the same concrete both to failure in compression and in the internal fracture test, thus eliminating the difference in strength between reference cubes and the specimens. This difference can be substantial, especially with crushed-rock aggregates and sands. It is considered that the number of tests recommended is sufficient to assess the compressive strength of structural members in situ. It is open to the engineer concerned to consider the use of more tests; however, we suggest that the law of diminishing returns will be operating and the cost of additional tests is likely to outweigh the increase in accuracy of the mean of sampling tests. The accuracy of prediction of compressive strength of concrete depends upon the scatter of points around the calibration curve, i.e. the width of the confidence limit band.

\section{REFERENCES}

1. BUNGEY, J. H. Determining concrete strength by using smalldiameter cores. Magazine of Concrete Research. Vol. 31, No. 107. June 1979. pp. $91-98$.

2. BUnGeY, J. H. Concrete strength determination by pull-out tests on wedge-anchor bolts. Proceedings of the Institution of Civil Engineers. Part 2: Research and Theory. Vol. 71. June 1981. pp. 379-394. 